Pengpeng Cheng*, Daoling Chen and Jianping Wang

\title{
Research on prediction model of thermal and moisture comfort of underwear based on principal component analysis and Genetic Algorithm-Back Propagation neural network
}

\author{
https://doi.org/10.1515/ijnsns-2020-0068 \\ Received March 27, 2020; accepted September 25, 2020; published online October 21, 2020
}

\begin{abstract}
In order to improve the efficiency and accuracy of thermal and moisture comfort prediction of underwear, a new prediction model is designed by using principal component analysis method to reduce the dimension of related variables and eliminate the multi-collinearity relationship between variables, and then inputting the converted variables into genetic algorithm (GA) and BP neural network. In order to avoid the problems of slow convergence speed and easy falling into local minimum of Back Propagation (BP) neural network, this paper adopted GA to optimize the weights and thresholds of BP neural network, and utilized MATLAB software to program, and established the prediction models of BP neural network and GA-BP neural network. To verify the superiority of the model, the predicted result of GA-BP, PCA-BP and BP are compared with GA-BP neural network. The results show that PCA could improve the accuracy and adaptability of GA-BP neural network for thermal and moisture comfort prediction. PCA-GA-BP model is obviously superior to GA-BP, PCA-BP, BP, SVM and K-means prediction models, which could accurately predict thermal and moisture comfort of underwear. The model has better accuracy prediction and simpler structure.
\end{abstract}

Keywords: Genetic Algorithm-Back Propagation (GA-BP) neural network; male; principal component analysis; thermal and moisture comfort; underwear.

\section{Introduction}

Thermal and moisture comfort has always been a research hot spot in the field of clothing, and thermal and wet is also a key factor that cannot be ignored for human body function or health. High temperature and humidity are not conducive to the thermal balance of the body. If human body does not dissipate heat in time, high temperature will easily lead to the decline of human muscle function and fatigue. Increased humidity will prevent sweat from evaporating and destroy heat balance. However, thermal and moisture is embodied in clothing, which is inseparable and coexist. That is, there is a subtle relationship between the influencing factors of thermal and wet comfort, which affects each other and is non-linear. For example, some heat will be taken away in the process of moisture permeability, and some steam will be taken away in the process of heat conduction of clothing. Therefore, the thermal comfort or wet comfort cannot be studied solely for the study of thermal and wet comfort. In view of this phenomenon, GA-BP algorithm is used to systematically study the thermal and moisture comfort of underwear.

There are various algorithms applied to evaluate the comfort of underwear or underwear fabrics. Kong et al., Huang et al. and Chen et al. [1-3] used grey theory to analyse the thermal and moisture comfort, and

\footnotetext{
*Corresponding author: Pengpeng Cheng, College of Fashion and Design, Donghua University, Shanghai, China, E-mail: cppcdl3344@163.com

Daoling Chen, Clothing and Design Faculty, Minjiang University, Fuzhou, China

Jianping Wang, College of Fashion and Design, Donghua University, Shanghai, China; and Key Laboratory of Clothing Design and Technology, Donghua University, Ministry of Education, Shanghai, China
}

Ә Open Access. ( 2020 Pengpeng Cheng et al., published by De Gruyter. $(\underset{\text { BY }}{\mathrm{BY}}$ This work is licensed under the Creative Commons Attribution 4.0 International License. 
different conclusions are drawn that the thickness and total tightness have different effects on the moisture permeability of fabrics. Wang et al. [4] adopted SPSS to evaluate the thermal comfort of women's jackets. Wang and Jing $[5,6]$ utilized artificial neural network, which is used to predict the thermal and moisture comfort of fabrics. Wang and Cui $[7,8]$ proposed fuzzy mathematics to evaluate the thermal and moisture comfort, contact comfort and other related properties of blended knitted fabrics.

This paper summarizes the research on thermal and moisture comfort of clothing, it is found that most of their studies use sweat thermal manikin, which cannot truly reflect thermal comfort, especially for the comfort study of underwear, which is only limited to the experimental of fabric or only underwear, and did not consider thermal and moisture in wearing state. At the same time, these studies only consider the fabric or the thickness of air, and do not consider the factors of human body shape.

Grey relational grade method, fuzzy mathematics, SPSS and BP algorithms are not accurate and applicable to this multi-factor coupling research result, which has certain limitations. Therefore, in order to optimize the test data and reflect the authenticity of the experiment, this paper proposed to design a new experimental scheme, used PCA to reduce the dimension of the characteristic parameters, removed irrelevant indexes and indexes with correlation, and avoided the redundancy of input characteristic values, thus reducing the calculation workload of GA-BP algorithm and simplifying the network structure. At the same time, the simplified data still retains most of the information of the original data. Genetic Algorithm (GA) has strong global search capability [9, 10], Back Propagation (BP) neural networks have strong non-linear fuzzy approximation ability [11-13], the combination of GA and BP is used to evaluate thermal and moisture comfort, and the results are more scientific and credible.

\section{Experiment}

\subsection{Selection of testers}

The participants were five young men, all of whom were healthy. Before the experiment, the participants were asked not to smoke, drink alcohol and take stimulating drugs. Tell them the process and danger of the test truthfully, but don't tell the subjects about the test fabric, so as to prevent the physiological and psychological influences of the subjects. The body size of the five college students are in Table 1.

\subsection{Experimental condition}

(1) All the experiments were carried out in an artificial climate chamber with constant temperature and humidity. The ambient temperature was $(25 \pm 2)^{\circ} \mathrm{C}$, the relative humidity was $(65 \pm 5) \%$ and the wind speed $\leq 0.1 \mathrm{~m} / \mathrm{s}$.

(2) Select men's underwear with XL size and the same style and different fabrics.

Table 1: Figure data of testers.

\begin{tabular}{lrrrrrrrrrr}
\hline No. & Age & Ht/cm & Wc/cm & Hc/cm & Tc/cm & Hh/cm & Whd/cm & Hat/cm & sH & BMI \\
\hline B1 & 22 & 170.1 & 73.9 & 84.7 & 51.3 & 84.2 & 14.3 & 1.5 & 1.13 & 22.7 \\
B2 & 24 & 172 & 77.5 & 99.2 & 45.7 & 86.4 & 20.3 & 2.8 & 1.24 & 21.7 \\
B3 & 23 & 170.3 & 75.1 & 90.6 & 47.1 & 85.2 & 13.3 & 2.1 & 1.01 & 21.1 \\
B4 & 25 & 173.4 & 76 & 82.5 & 45.4 & 86.7 & 10.6 & 3.2 & 1.21 & 21.8 \\
B5 & 22 & 175.5 & 80.7 & 99.1 & 54.1 & 85 & 17.2 & 4.6 & 1.33 & 22.1 \\
Mean & 23 & 172.3 & 76.6 & 91.2 & 48.7 & 85.5 & 15.1 & 2.8 & 1.18 & 21.9 \\
\hline
\end{tabular}

Ht-Height; Wc-Waist circumference; Hc-Hip circumference; Tc-Thigh circumference; Hh-Hip height; Whd-Waist-hip height difference; Hat-Hip-abdomen thickness; sH-Sagittal diameter ratio of Hip. 
(3) Before each experiment is carried out, ensure that all experimental underpants are restored to their original state and placed in the artificial climate room for $24 \mathrm{~h}$.

(4) The tester randomly selects and tries on each underwear in turn without being informed of the underwear fabric for the experiment.

(5) During measurement, the upper body of the tester is exposed, and each link is measured 3 times. In the next measurement, it shall be taken off and put on again before measurement.

\subsection{Experimental underwear}

Fifty underwears of different brands were purchased randomly. Take 10 underwears as an example. Table 2 shows the fabric parameters of underwear.

\subsection{Subjective comfort evaluation}

Five testers entered the artificial climate room at the same time on different days to prepare according to the experimental requirements. The whole test process is as follows: preparation (20 $\mathrm{min}$, aimed at adapting to the test environment) $\rightarrow$ sit quietly $(15 \mathrm{~min}) \rightarrow \operatorname{limb}$ movement $(10 \mathrm{~min}) \rightarrow$ rest $(5 \mathrm{~min}) \rightarrow$ jogging on treadmill $(5 \mathrm{~min}$, speed controlled at $4.6 \mathrm{~km} / \mathrm{h}) \rightarrow$ rest $(15 \mathrm{~min})$. Each participant wore all experimental underwear in turn and conducted experiment according to the above procedure. At the same time, the staff should record the subjective comfort evaluation value of the participants in each experimental link, and finally calculate the average value as the final subjective evaluation.

According to ISO 10551-2001, "Ergonomics of Thermal Environment Evaluates Impact of Thermal Environment by Subjective Judgment Scale", subjective evaluation is carried out, as shown in Figure 1 and Table 3.

\subsection{Kendall correlation coefficient (KCC) test of subjective evaluation results}

In order to verify the consistency of the evaluation results among the five evaluators (i.e., five testers), Kendall is used to analyse whether the judgement criteria of the evaluators are consistent and fair. KCC, also known as harmony coefficient, is a method to express the correlation degree of multi-rank variables.

$$
k=\frac{12 \sum_{i=1}^{\beta} R_{i}^{2}-3 \cdot \alpha^{2} \beta(\beta+1)^{2}}{\alpha^{2} \cdot \beta \cdot\left(\beta^{2}-1\right)}
$$

where $\alpha$ is the number of judges or testers; $\beta$ is the number of observation objects or observation indicators and $R_{i}$ is the sum of the ranks of the $i$ observation object.

It is generally believed that Kendall coordination coefficient $<0.2$, it indicates poor consistency. Between 0.2 and 0.4 , the consistency degree is general. The consistency between 0.4 and 0.6 is moderate. Between 0.6 and 0.8 , the consistency degree is strong. Between 0.8 and 1.0, the consistency is very strong.

The original hypothesis Kendall coefficient is 0, i.e., completely inconsistent. As shown by the abovementioned test, asymptotics significance value $=0.000<0.05$. The original hypothesis is not valid (obviously not equal to zero), whereas it shows Kendall consistency.

Kendall correlation coefficient $\left(\right.$ Kendall $\mathrm{W}^{\mathrm{a}}$ ) $=0.924$ (see Table 4), which is greater than 0.8 , indicating that the comfort evaluation of underwear by five professionals has a high consistency level and the data are reliable. 


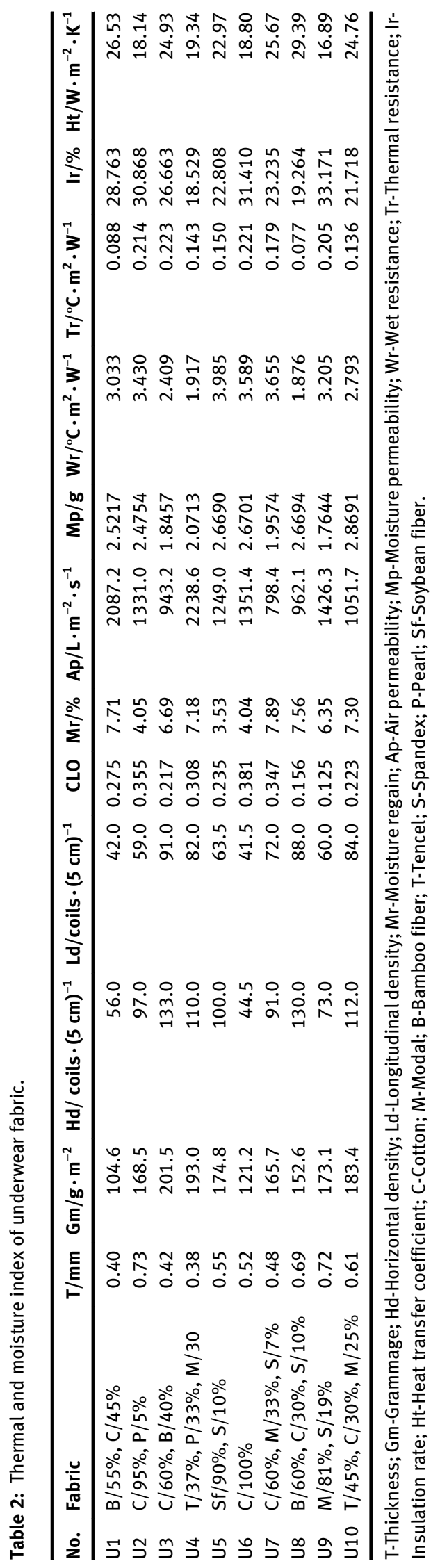




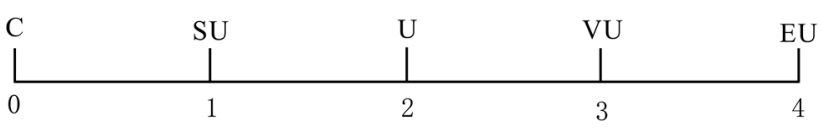

Figure 1: Subjective judgement scale. Note: level 0 (comfortable) (C), level 1 (slightly uncomfortable (SU), level 2 (uncomfortable) (U), level 3 (very uncomfortable) (VU), and level 4 (extremely uncomfortable) (EU).

Table 3: Subjective evaluation values of heat and moisture comfort in wearing state.

\begin{tabular}{lcccccccccc}
\hline & U1 & U2 & U3 & U4 & U5 & U6 & U7 & U8 & U9 & U10 \\
\hline B1 & 1.0 & 1.6 & 1.1 & 0.8 & 1.0 & 1.7 & 0.8 & 1.1 & 0.7 & 1.0 \\
B2 & 1.1 & 1.4 & 1.2 & 0.6 & 1.2 & 1.6 & 0.9 & 0.9 & 0.7 & 1.1 \\
B3 & 0.9 & 1.5 & 1.2 & 0.6 & 1.1 & 1.6 & 0.7 & 1.1 & 0.8 & 1.1 \\
B4 & 0.9 & 1.6 & 1.1 & 0.7 & 1.2 & 1.5 & 0.9 & 1.0 & 0.6 & 1.3 \\
B5 & 0.9 & 1.7 & 1.1 & 0.8 & 1.2 & 1.6 & 0.9 & 0.9 & 0.8 & 1.1 \\
Mean & 1.0 & 1.6 & 1.1 & 0.7 & 1.1 & 1.6 & 0.8 & 1.0 & 0.7 & 1.1 \\
\hline
\end{tabular}

Table 4: Inspection statistics.

\begin{tabular}{lr}
\hline$N$ & 5 \\
Kendall $W^{\mathrm{a}}$ & 0.924 \\
Chi-square & 41.572 \\
Df & 9 \\
Asymptotics significance & 0.000 \\
\hline
\end{tabular}

${ }^{\mathrm{a} K e n d a l l ~ S y n e r g y ~ c o e f f i c i e n t . ~}$

\section{PCA-GA-BP analysis of heat and moisture comfort data and establishment of model}

\subsection{PCA optimization of input parameters}

The initial input parameters of GA-BP are those in Tables 1 and 2, which may lead to network redundancy due to too many input parameters and complicated correlation. Multiple collinearities between input variables will lead to strange changes in network parameters, thus reducing the generalization performance of the network. Therefore, PCA is used to reduce the dimension of parameters, eliminate some irrelevant factors, and at the same time, eliminate the multi-collinearity among variables to improve the accuracy of GA-BP model.

(1) Input optimization of underwear parameters

Principal component analysis was carried out on Table 1 by SPSS. Before principal component analysis, KMO and Bartlett tests were carried out on 12 items of data such as thickness, gram weight and longitudinal density. KMO value is an indicator used to check whether the data can be used for principal component analysis, as shown in Table 5.

Table 5: Inspection standards for principal component analysis.

\begin{tabular}{lc}
\hline Degree of suitability for principal component analysis & KMO value range \\
\hline Very suitable & KMO > 0.9 \\
Suitable for & $0.9>\mathrm{KMO}>0.8$ \\
General & $0.8>\mathrm{KMO}>0.7$ \\
Not very suitable & $0.7>\mathrm{KMO}>0.6$ \\
Not suitable & $0.6>\mathrm{KMO}$ \\
\hline
\end{tabular}


As can be seen from Table 6 , KMO value $=0.835$, indicating that parameter data of underwear can be factor analysed. Then, the input parameters of underwear are analysed by principal component analysis, as shown in Tables 7 and 8 .

It can be seen from Table 7 that the cumulative contribution rate of principal components with eigenvalues greater than one is $84.306 \%$, so five key feature size parameters of human body are obtained, which can be considered as sufficient to reflect the information of the original measurement data.

Table 6: Inspection of KMO and Bartlett.

Kaiser-Meyer-Olkin metric for sampling adequacy

Table 7: Expositive the total variance.

\begin{tabular}{|c|c|c|c|c|c|c|c|c|c|}
\hline \multirow[t]{2}{*}{ Component } & \multicolumn{3}{|c|}{ Initial eigenvalue } & \multicolumn{3}{|c|}{ Select sum of squares and load } & \multicolumn{3}{|c|}{ Rotational sum of squares and load } \\
\hline & Total & Variance \% & Cumulative \% & Total & Variance \% & Cumulative \% & Total & Variance \% & Cumulative \% \\
\hline 1 & 3.695 & 30.793 & 30.793 & 3.695 & 30.793 & 30.793 & 2.612 & 21.764 & 21.764 \\
\hline 2 & 2.734 & 22.784 & 53.576 & 2.734 & 22.784 & 53.576 & 2.249 & 18.746 & 40.510 \\
\hline 3 & 1.769 & 14.743 & 68.319 & 1.769 & 14.743 & 68.319 & 2.116 & 17.635 & 58.145 \\
\hline 4 & 1.351 & 11.262 & 79.581 & 1.351 & 11.262 & 79.581 & 1.908 & 15.902 & 74.047 \\
\hline 5 & 1.013 & 8.439 & 88.020 & 1.013 & 8.439 & 88.020 & 1.677 & 13.973 & 88.020 \\
\hline 6 & 0.797 & 6.641 & 94.661 & & & & & & \\
\hline 7 & 0.273 & 2.278 & 96.938 & & & & & & \\
\hline 8 & 0.214 & 1.786 & 98.724 & & & & & & \\
\hline 9 & 0.131 & 1.088 & 99.812 & & & & & & \\
\hline 10 & 0.023 & 0.188 & 100.000 & & & & & & \\
\hline 11 & $8.605 \mathrm{E}-16$ & $7.170 \mathrm{E}-15$ & 100.000 & & & & & & \\
\hline 12 & $-3.904 \mathrm{E}-17$ & $-3.253 \mathrm{E}-16$ & 100.000 & & & & & & \\
\hline
\end{tabular}

Table 8: Rotating component matrix.

\begin{tabular}{|c|c|c|c|c|c|}
\hline & & & & & Component \\
\hline & 1 & 2 & 3 & 4 & 5 \\
\hline Moisture regain & 0.943 & & & & \\
\hline Insulation rate & -0.728 & & & & \\
\hline Grammage & 0.634 & & & & \\
\hline Thermal resistance & -0.612 & & . & & \\
\hline Air permeability & & -0.875 & & & \\
\hline Moisture permeability & & 0.759 & & & \\
\hline Heat transfer coefficient & & 0.678 & & & \\
\hline Horizontal density & & & 0.928 & & \\
\hline Thickness & & & -0.808 & & \\
\hline CLO & & & & 0.942 & \\
\hline Longitudinal density & & & & -0.805 & \\
\hline Wet resistance & & & & & 0.920 \\
\hline
\end{tabular}


From Table 8, it can be seen that the input indexes of underwear parameters: the first main components are moisture regain, insulation rate, grammage and thermal resistance. The second principal component is air permeability, moisture permeability and heat transfer coefficient, and the third principal component is horizontal density and thickness. The fourth principal component is CLO value, longitudinal density. The fifth main component is wet resistance. Through principal component analysis, according to relevant articles [14-18] and professional knowledge, moisture regain, insulation rate, air permeability, horizontal density, CLO value and moisture permeability are selected as the input of underwear parameters. Therefore, the main fabric indexes that reflect the thermal and wet comfort of underwear are moisture regain, insulation rate, air permeability, horizontal density, CLO value and wet resistance.

(2) Input optimization of body shape parameter

According to relevant documents and my previous research [19-22], this paper mastered the key parts that affect the body shape or which parts have a greater correlation, so this paper finally selected abdominal and hip thickness, BMI, waist circumference, thigh circumference, waist-hip height as the input parameters of the body shape.

\subsection{Pattern design of GA-BP algorithm}

First, the BP algorithm is adopted to select initial values through a certain number of studies. Secondly, GAm is used to optimize the weights and thresholds of the BP network, to avoid the defect that the BP network falls into local optimization when optimizing the weights and thresholds, and completing the network learning with given precision [23]. When the GA has a slow convergence speed, $\mathrm{BP}$ algorithm is adopted to complete the network learning with a given precision. This network model has the advantages of strong generalization, good stability, strong memory, global convergence and fast speed. Figure 2 shows its prediction flow.

(1) Parameter setting:base on BP neural network, GA is used to optimize the weight and threshold of BP algorithm to improve the accuracy of BP algorithm. Its flow chart is shown in Figure 2. Six characteristic variables (moisture regain, heat preservation rate, air permeability, horizontal density, Crohn's value and wet resistance) and five variables (abdominal hip thickness, BMI, waist circumference, thigh circumference and waist hip height) of the fabric are taken as input layers of the artificial neural network. Since the subjective evaluation is consistent, the body shape data corresponding to each underwear fabric parameter can be randomly selected from five evaluators, and the subjective evaluation value of the thermal and moisture comfort of the underwear is the subjective evaluation value of the evaluator, so the output value is the average value of the subjective evaluation of each underwear.

The hidden layer is $\sqrt{I+O}+\partial$, where I is the number of input layer variables (i.e., the number of input layer nodes or neurons) and $\mathrm{O}$ is the number of output layer variables (i.e., the number of output layer nodes or neurons), which is a constant between 1 and 10. According to some studies [24-26], the hidden layer is usually $2-15$, and this item is selected as 13 . The output layer is subjective comfort value. Then, the network structure this time is 11-13-1. In addition, the maximum number of this training is set at 1000 and the learning rate is 0.1 . The minimum learning error is 0.00001 and the additional momentum factor net.trainParam.mc $=0.95$; minimum performance gradient net.trainParam.min_grad = 1e-6; Genetic neural parameter evolution algebra, i.e., iteration number maxgen $=30$; sizepop $=50$; cross probability pcross $=0.4$; probability of mutation: pmutation $=0.2$. 


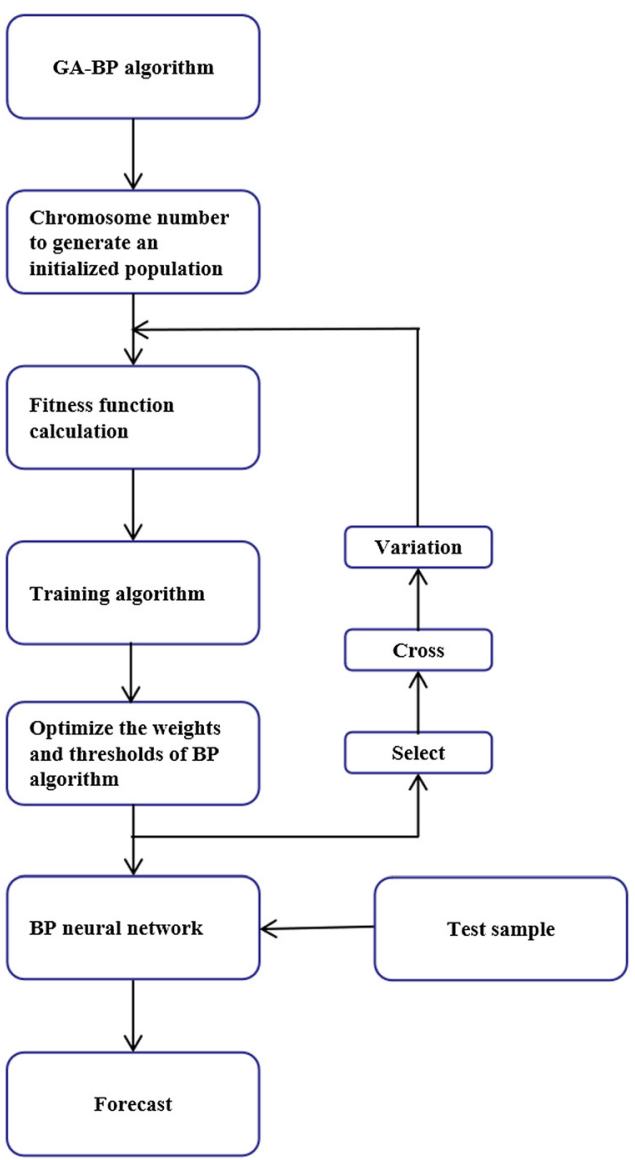

Figure 2: GA-BP prediction structure diagram.

(2) Normalization processing

Because the magnitude and dimension of these parameter values are different, there will be great differences between the data, so they should be standardized before establishing the model. In this paper, Formula (2) is directly adopted to normalize or normalize the data of $\mathrm{X}$ matrix.

$$
x_{i j}^{*}=\frac{\left(2 \times x_{i j}-x_{i \min }\right)}{x_{i \max }-x_{i \min }}-1
$$

where $x_{i j}$ is the value before normalization of column $j$ of the sample in row $i ; x_{i j}{ }^{*}$ is the normalized value of column $j$ of the sample in row $i ; x_{\operatorname{imax}}$ and $x_{\text {imin }}$ are the maximum and minimum values before normalization, respectively.

(3) Transfer function: Log-Sigmoid function is used for transfer function from input layer to hidden layer and transfer function from hidden layer to output layer.

$$
\log \operatorname{sig}(x)=\frac{1}{1+e^{-x}}
$$

(4) Calculation of fitness function.

The coded chromosome is taken as the initial population of GA algorithm, and the weights and thresholds of BP training set are optimized according to fitness function (4). 
Table 9: Comparison of prediction models.

\begin{tabular}{lrrrrrrrrr}
\hline No. & Subjective evaluation value & PCA-GA-BP & Error & GA-BP & Error & PCA-BP & Error & BP & Error \\
\hline U1 & 1 & 1.0395 & 0.0395 & 1.0664 & 0.0664 & 0.7475 & -0.2525 & 1.2522 & 0.2522 \\
U5 & 1.1 & 1.0857 & -0.0143 & 1.1006 & 0.0006 & 0.6468 & -0.4532 & 1.2715 & 0.1715 \\
U9 & 0.7 & 0.6236 & -0.0764 & 0.5545 & -0.1455 & 0.6706 & -0.0294 & 1.0493 & 0.3493 \\
U20 & 1.7 & 1.7486 & 0.0486 & 1.8488 & 0.1488 & 1.2509 & -0.4491 & 1.2116 & -0.4884 \\
U37 & 0.9 & 0.9210 & 0.0210 & 0.9898 & 0.0898 & 0.6884 & -0.2116 & 1.1661 & 0.2661 \\
U41 & 1.4 & 1.4315 & 0.0315 & 1.4861 & 0.0861 & 1.2972 & -0.1028 & 1.0691 & -0.3309 \\
U49 & 1.1 & 1.0429 & -0.0571 & 1.0029 & -0.0971 & 1.5636 & 0.4636 & 1.1101 & 0.0101 \\
Maximum absolute error & & & 0.0764 & & 0.1488 & & 0.4636 & & 0.4884 \\
Average error & & 0.0412 & & 0.0906 & & 0.2803 & & 0.2669 \\
\hline
\end{tabular}

Table 10: Convergence situation of prediction models.

\begin{tabular}{lrr}
\hline Models & Epochs & Times/s \\
\hline PCA-GA-BP & 122 & 57 \\
GA-BP & 301 & 98 \\
PCA-BP & 333 & 103 \\
BP & 625 & 172 \\
\hline
\end{tabular}

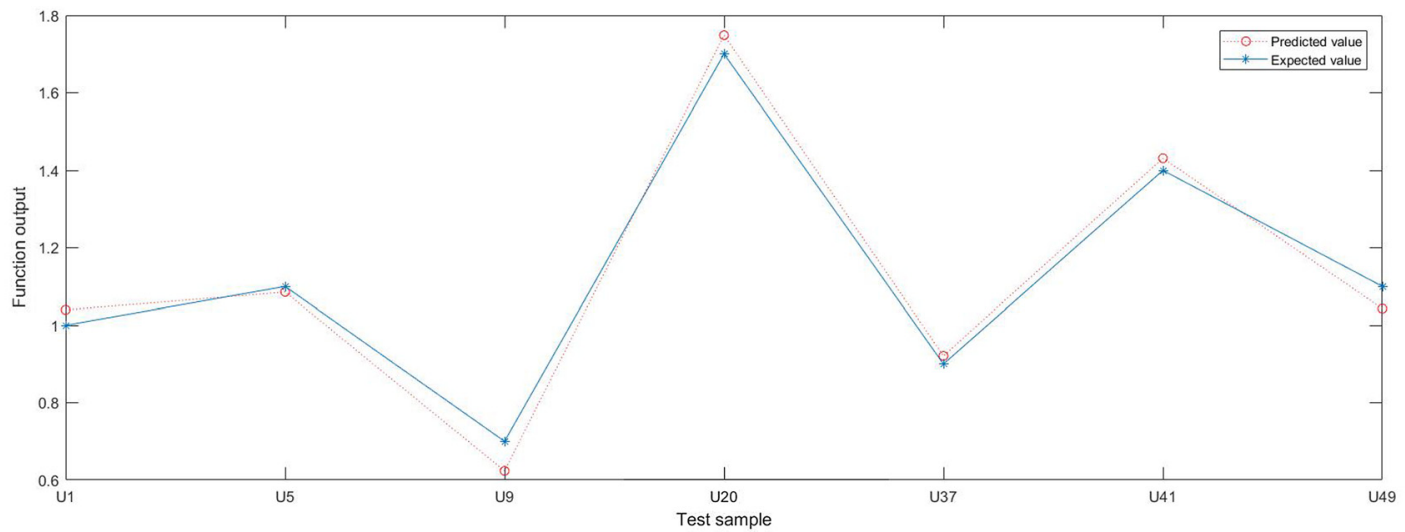

(a) predicted and expected values

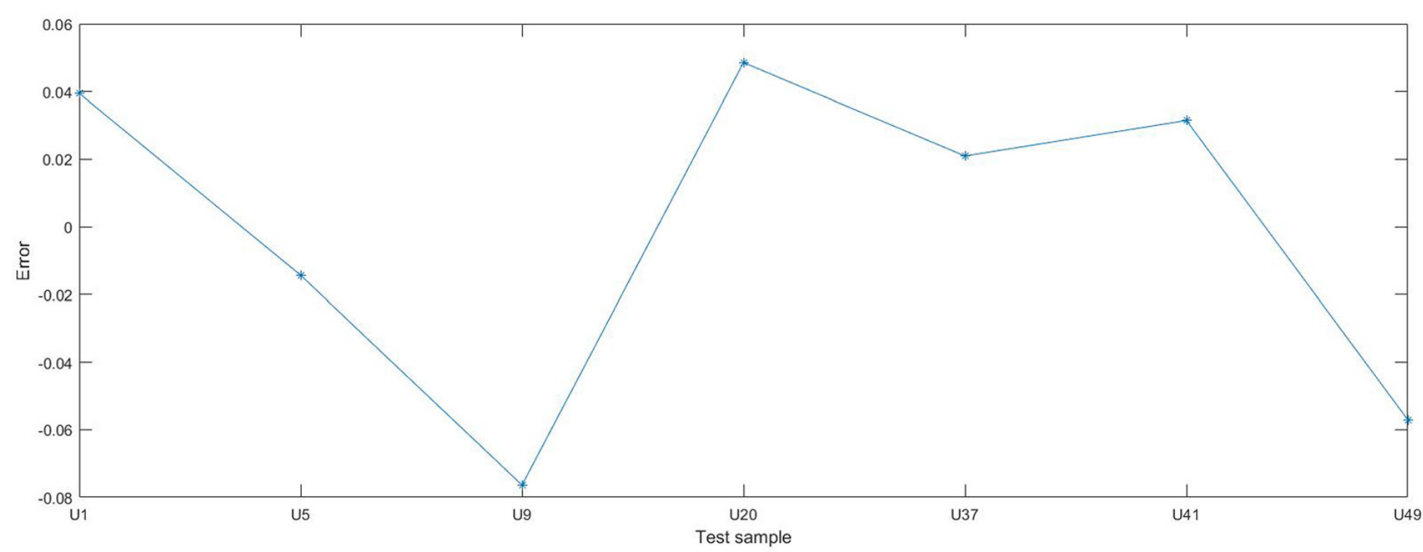

(b) prediction error

Figure 3: PCA-GA-BP model. 


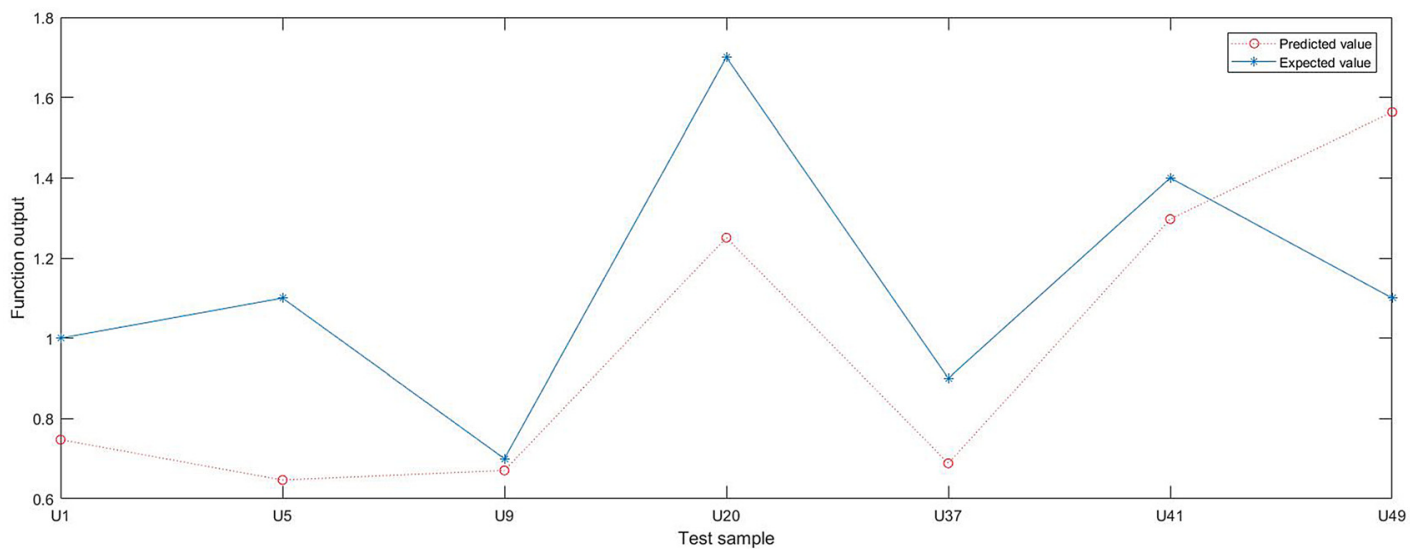

(a) predicted and expected values

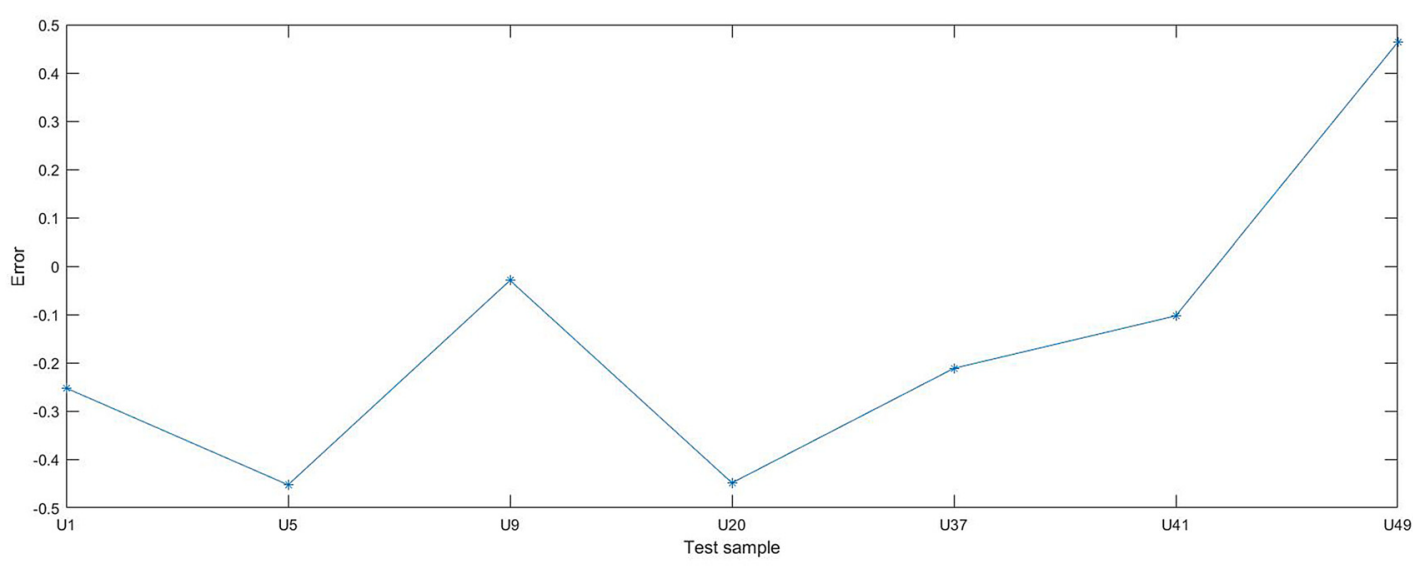

(b) prediction error

Figure 4: PCA-BP model.

$$
F=\frac{1}{\sum_{i=1}^{5}\left(C_{i}^{\prime}-C_{i}\right)^{2}}
$$

where $C_{i}^{\prime}(i=1,2,3,4,5)$ is the fitting value of BP algorithm; $C_{i}(i=1,2,3,4,5)$ is the actual value of subjective evaluation of comprehensive comfort.

(5) Test samples and training samples.

U1, U5, U9, U20, U37, U41 and U49 were randomly selected as test samples, and the remaining 43 groups of data were used as training samples.

\section{Results and discussion}

In order to verify the feasibility and effectiveness of the genetic neural network of the principal component analysis, the prediction results of PCA-GA-BP model are compared with GA-BP, PCA-BP and BP models, as shown in Tables 9 and 10, Figures 3-6.

As can be seen from Tables 9 and 10 and Figures 3-6, PCA-GA-BP prediction accuracy is better than GABP, PCA-BP and BP. The prediction errors of PCA-GA-BP are all less than 0.08 , the maximum error is only 0.0764 , the average error is 0.0412 , the maximum error of GA-BP is only 0.1488 , the average error is 0.0906 , which shows that GA in GA-BP model has better global search performance and reduces the possibility of BP 


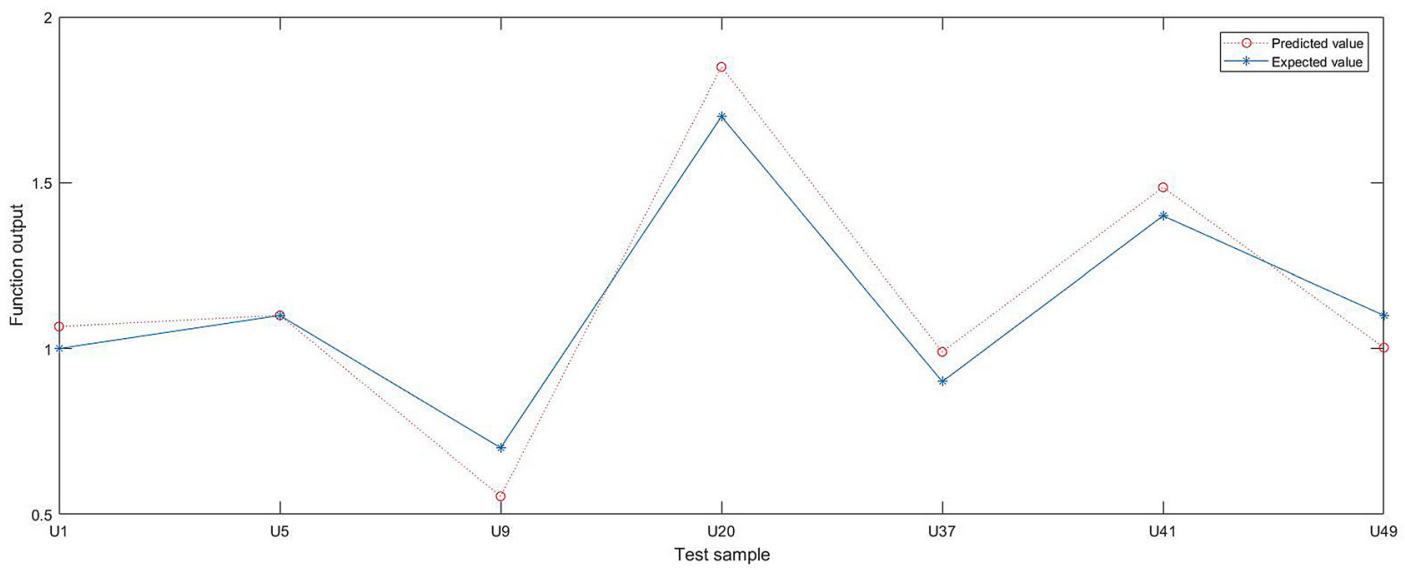

(a) predicted and expected values

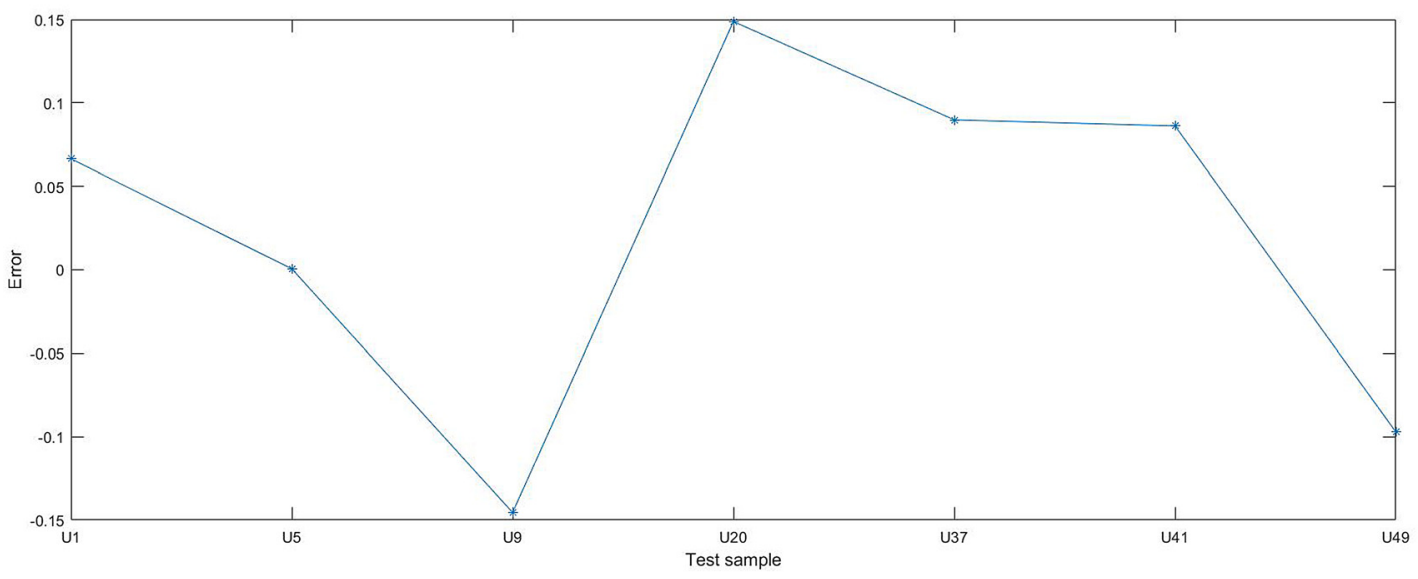

(b) prediction error

Figure 5: GA-BP model.

falling into local extremum, GA can well deal with the correlation and non-linear problems between indexes, and PCA-GA-BP has higher prediction accuracy than GA-BP, which indicates that PCA improves the prediction accuracy of GA-BP to a certain extent. Table 10 shows that the convergence speed of PCA-GA-BP model is faster than other models and takes the least time. Epochs of PCA-GA-BP model is 122 and the time taken is $57 \mathrm{~s}$. Epochs of GA-BP model is 301 and the time taken is $98 \mathrm{~s}$, which PCA-BP is close to. Epochs and time of $\mathrm{BP}$ are the most of them.

From Tables 9 and 10, it can be seen that the prediction effect of BP neural network is the worst, which shows that the strong correlation and non-linearity between input parameters without dimensionality reduction, and the weakness that $\mathrm{BP}$ is easy to fall into the local optimal solution, result in the lower prediction accuracy of BP. PCA-GA-BP model could effectively reduce the scale of input data and has better training speed, which overcomes the shortcomings of slow convergence speed and long training time of BP. This shows that the reduction of input data dimension is beneficial to the optimization iteration of neural network parameters, and makes parameter changes more concise and effective.

The maximum error and average error of PCA-BP and BP are close, and the error is higher than that of PCA-GA-BP and GA-BP, which shows that although PCA has less output parameters to some extent and achieves dimension reduction effect, the effect on BP neural network which is prone to fall into local optimal solution is not obvious. Therefore, PCA should be combined with other neural networks to optimize BP and achieve higher prediction accuracy. Therefore, the results show that PCA-GA-BP is reliable in predicting subjective evaluation values of thermal and moisture comfort. 


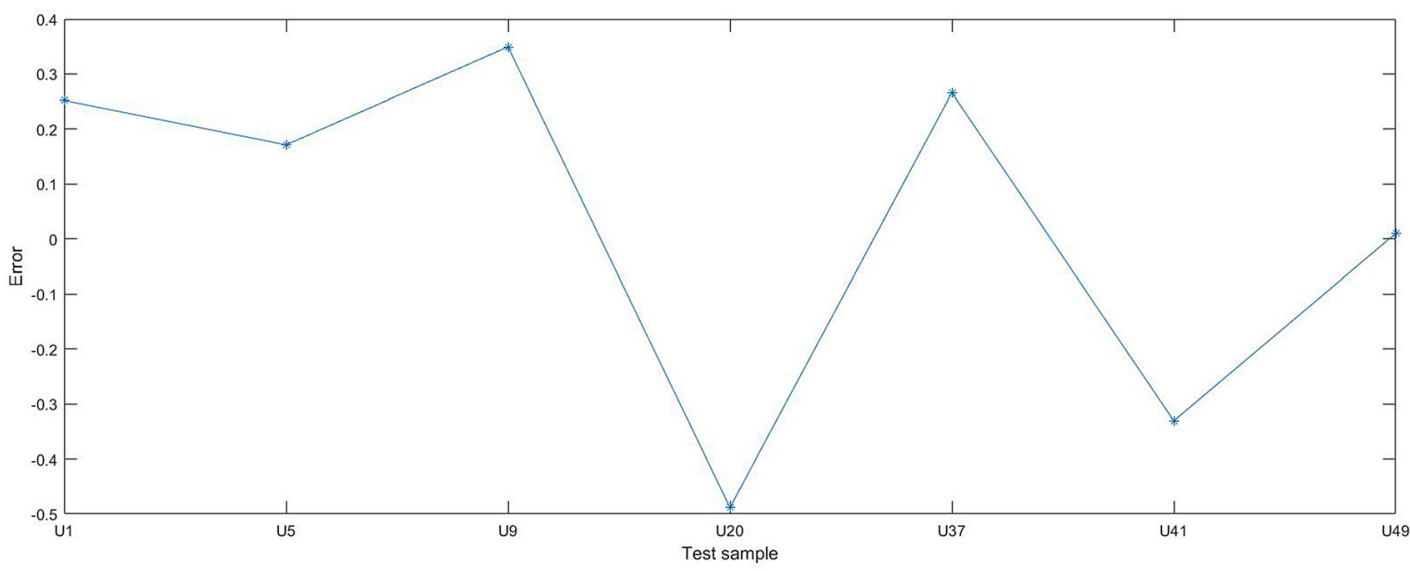

(a) predicted and expected values

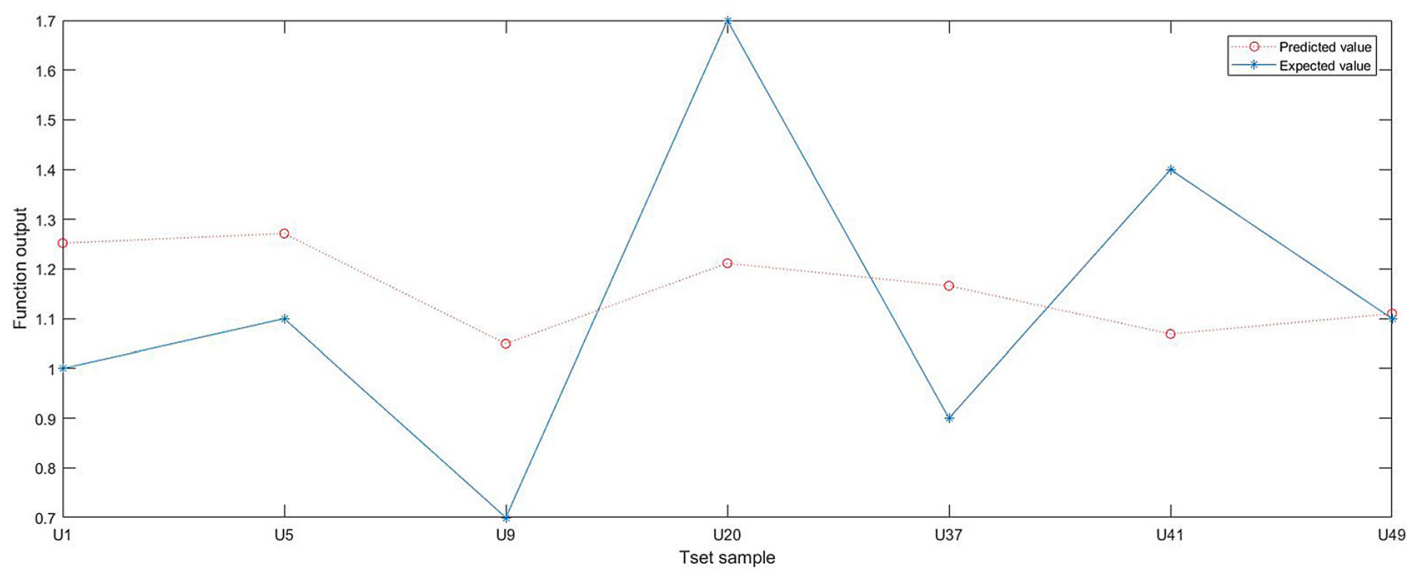

(b) prediction error

Figure 6: BP model.

\section{Conclusion}

(1) There have been many researches on the thermal and moisture comfort of human body, but there are some limitations due to various reasons. In this paper, the human body shape index and the parameters underwear fabric are used as the prediction indexes, and the obtained experimental data are more authentic and reliable.

(2) There are many factors affecting thermal and moisture comfort, and the relationship between them is highly non-linear and complex. The effect is not obvious with common algorithm, while PCA-GA-BP model could deal with this relationship well.

(3) The prediction effect of PCA-GA-BP model is close to the actual value, which shows that prediction of subjective evaluation by PCA-GA-BP model for thermal and moisture comfort is reasonable and reliable, so the model is universal and should be popularized.

Acknowledgment: The authors would like to acknowledge the financial support from the Fundamental Research Funds for the Central Universities (2232020G-08) and National Key Research and Development Plan "Science and Technology in Winter Olympic Games" (2019YFF0302100).

Author contribution: All the authors have accepted responsibility for the entire content of this submitted manuscript and approved submission. 
Research funding: This study was financially supported by China Scholarship Council and Fujian Province Social Science Planning Project (FJ2020C049) and the Fundamental Research Funds for the Central Universities (2232020G08) and national key research and development plan "science and technology in Winter Olympic Games” (2019YFF0302100).

\section{References}

[1] L. J. Kong and X. Yan, "Application of gray system theory in evaluation of thermal and moisture comfort of bast fiber fabrics," J. Textil. Res., vol. 28, no. 4, pp. 41-44, 2007.

[2] S.P. Huang, C. Q. Ma, and H. S. Zhou, "Model of thermal and moisture comfort evaluation of bamboo fiber fabric based on grey correlation degree,” J. Textil. Res., vol. 30, no. 9, pp. 33-36, 2009.

[3] X. Y. Chen, Y. Ban, and W. Wu, "Gray theory applied to forecasting comfortableness of fabrics against heat and moisture," J. Inn. Mong. Univ. Technol. (Soc. Sci. Ed.), vol. 30, no. 2, pp. 150-154, 2011.

[4] Y. Y. Wang, J. H. Pan, and S. Y. Liu, "Study on the relation between the air gap size under clothing and the comfort sensations," J. Donghua Univ., vol, 23, no. 5, pp. 101-107, 2006.

[5] L. Y. Wang and L. B. Wang, “Discussion about artificial neural network predicting clothing and fabrics comfort," J. Qingdao Univ. (Nat. Sci. Ed.), vol. 21, no. 1, pp. 82-85, 2006, 91.

[6] X. N. Jing and Y. B. Li, "Study on heat-moisture comfort evaluation index of fabrics with grey theory," Melliand China, vol. 39 , no. 4, pp. 70-72, 2011, 74-75.

[7] F. Wang, Study on Comfort of PTT Blended Knitted Fabric for Underwear, Suzhou, Soochow University, 2012.

[8] D. D. Cui, Development and Comfort Properties of Milk Fiber Blended Knitted Underwear Fabric, Shanghai, Donghua University, 2015.

[9] L. C. Chang, F. J. Chang, and H. C. Hsu, "Real-time reservoir operation for flood control using artificial intelligent techniques," Int. J. Nonlinear Sci. Numer. Stimul., vol. 11, no. 11, pp. 887-902, 2010.

[10] H. R. Moetamedzadeh, E. Khanmirza, and R. Madoliat, "Transient simulation of natural gas network by hybrid taguchi binary genetic algorithm," Int. J. Nonlinear Sci. Numer. Stimul., vol. 21, no. 1, pp. 51-63, 2020.

[11] T. Sunil and S. K. Panigrahi, “A hybrid genetic algorithm and back-propagation classifier for gearbox fault diagnosis,” Appl. Artif. Intell., vol. 31, nos 7-8, pp. 593-612, 2017.

[12] Y. J. Liang, C. Ren, H. Y. Wang, Y. B. Huang, and Z. T. Zheng, "Research on soil moisture inversion method based on GA-BP neural network model," Int. J. Rem. Sens., vol. 40, nos 5-6, pp. 2087-2103, 2019.

[13] T. Kerh, Y. L. Chan, and D. Gunaratnam, "Treatment and assessment of nonlinear seismic data by a genetic algorithm based neural network model,” Int. J. Nonlinear Sci. Numer. Stimul., vol. 10, no. 1, pp. 45-56, 2009.

[14] L. Y. Liu and L. Liu, "Subjective evaluation of clothing comfort and their correlation with laboratory test,"J. Qingdao Univ. (Nat. Sci. Ed.), vol. 28, no. 3, pp. 60-63, 2013, 68.

[15] M. Li, Comfort Evaluation and Moisture Transfer Performance Prediction of Knitted Fabrics Based on New Function, Shanghai, Donghua University, 2010.

[16] B. Z. Ke, Development of New Sports Fabrics and Establishment of Intelligent Prediction Model for Subjective Thermal and Moisture Comfort of Fabrics, Shanghai, Donghua University, 2006.

[17] X. Y. Duan, “Development and application of new thermal and moisture comfort underwear fabric," Jiangsu Textil., vol. 27, no. 3, pp. 50-52, 2008.

[18] F. F. Sun, J. Li, and J. S. Han, "Evaluation and prediction of clothing comfort based on fabric physical properties,"J. Textil. Res., vol. 27, no. 11, pp. 82-85, 2006.

[19] P. P. Cheng and D. L. Chen, "Research and analysis of 18-25-year-old adult body in Fujian," J. Wuhan Textil. Univ., vol. 29, no. 06, pp. 57-60, 2016.

[20] P. P. Cheng and D. L. Chen, "On the characteristics of Fujian adults under the transformation of garment industry," J. Cloth. Res., vol. 1, no. 04, pp. 369-374, 2016.

[21] Z. Q. Zhang, “Analysis of body shape of male college students in Central and Western China,”. Donghua Univ., vol. 37, no. 01, pp. 51-56, 2011.

[22] L. L. Hou, H. G. Wang, and W. B. Zhang, "Study on lower body shape of adult men in three areas,"J. Textil. Res., vol. 29, no. 10, pp. 95-97, 2008.

[23] W. Wang, M. Li, R. H. Hassanien, M. E. Ji, and Z. K. Feng, “Optimization of thermal performance of the parabolic trough solar collector systems based on GA-BP neural network model,” Int. J. Green Energy, vol. 14, no. 10, pp. 819-830, 2017.

[24] L. J. Kong and X. Yan, "Study on test of moisture permeability of fabric and application of matlab,"J. Hunan Inst. Eng. (Natural Sci. Ed.), vol. 15, no. 2, pp. 84-87, 2005.

[25] X. N. Jing, Study on Thermal and Moisture Comfort of New Fabric Based on BP Artificial Neural Network, Tianjin, Tianjin Polytechnic University, 2010.

[26] B. Sun and N. F. Jiang, “Application of thermal comfort index based on artificial neural network,"J. Civ. Architect. Environ. Eng., vol. 33, no. S1, pp. 130-133, 2011. 